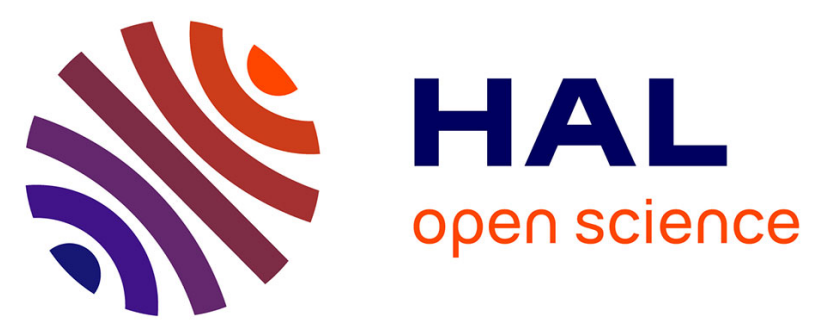

\title{
The role of surface diffusion in the growth mechanism of III-nitride nanowires and nanotubes
}

Bruno Daudin, Alexandra-Madalina Siladie, Marion Gruart, M. den Hertog, Catherine Bougerol, Benedikt Haas, Jean-Luc Rouvière, Eric Robin, Maria-José Recio-Carretero, Núria Garro, et al.

\section{To cite this version:}

Bruno Daudin, Alexandra-Madalina Siladie, Marion Gruart, M. den Hertog, Catherine Bougerol, et al. The role of surface diffusion in the growth mechanism of III-nitride nanowires and nanotubes. Nanotechnology, 2021, 32 (8), pp.085606. 10.1088/1361-6528/abc780 . hal-03429491

\section{HAL Id: hal-03429491 https://hal.science/hal-03429491}

Submitted on 15 Nov 2021

HAL is a multi-disciplinary open access archive for the deposit and dissemination of scientific research documents, whether they are published or not. The documents may come from teaching and research institutions in France or abroad, or from public or private research centers.
L'archive ouverte pluridisciplinaire HAL, est destinée au dépôt et à la diffusion de documents scientifiques de niveau recherche, publiés ou non, émanant des établissements d'enseignement et de recherche français ou étrangers, des laboratoires publics ou privés. 
This document is the unedited Author's version of a Submitted Work that was subsequently accepted for publication in Nanotechnology 32, 085606 (2020), copyright (c) Institute of Physics after peer review. To access the final edited and published work see [https://dx.doi.org/10.1088/1361-

\title{
The role of surface diffusion in the growth mechanism of III- nitride nanowires and nanotubes
}

\begin{abstract}
Bruno Daudin*1, Alexandra-Madalina Siladie ${ }^{1}$, Marion Gruart ${ }^{1}$, Martien den Hertog ${ }^{2}$, Catherine Bougerol ${ }^{2}$, Benedikt Haas ${ }^{3}$, Jean-Luc Rouvière ${ }^{3}$, Eric Robin ${ }^{3}$, Maria-José RecioCarretero ${ }^{4}$, Núria Garro ${ }^{4}$, Ana Cros $^{4}$
\end{abstract}

${ }^{1}$ Univ. Grenoble Alpes, CEA, IRIG-PHELIQS, NPSC, 17 rue des martyrs, 38000 Grenoble, France.

${ }^{2}$ Univ. Grenoble Alpes, CNRS, Grenoble INP, Institut Néel, 25 rue des martyrs, 38000 Grenoble, France.

${ }^{3}$ Univ. Grenoble Alpes, CEA, IRIG-MEM, LEMMA, 17 rue des martyrs, F-38000 Grenoble, France

${ }^{4}$ Institute of Materials Science (ICMUV), Universidad de Valencia, P.O. Box 22085, Valencia, Spain

*email: bruno.daudin@cea.fr

Abstract

The spontaneous growth of GaN nanowires in absence of catalyst is controlled by the Ga flux impinging both directly on the top and on the side walls and diffusing to the top. The presence of diffusion barriers on the top surface and at the frontier between the top and the sidewalls, however, causes an inhomogeneous distribution of $\mathrm{Ga}$ adatoms at the nanowire top surface resulting in a GaN accumulation in its periphery. The increased nucleation rate in the periphery promotes the spontaneous formation of superlattices in InGaN and AIGaN 
nanowires. In the case of AIN nanowires, the presence of $\mathrm{Mg}$ can enhance the otherwise short Al diffusion length along the sidewalls inducing the formation of AIN nanotubes.

\section{Introduction}

Semiconductor nanowires (NWs) and nanotubes (NTs) are a subject of increasing interest due to the remarkable physical properties of these nano-objects and to their potential applications ranging from a new class of light emitting devices (LEDs) to photovoltaic devices, photonics, nano-electronics and more, including single-NW devices. Thus, the eased elastic strain relaxation associated with a large free surface and aspect ratio [1] opens the path to the realization of long-wavelength LEDs with an increased In content in the active region [2,3]. In addition, the large surface to volume ratio of NWs makes them promising for sensor applications [4,5]. Semiconductor nanotubes (NTs), exhibiting even larger surface to volume ratio values, are expected to be suitable for such applications. For instance, it was theoretically pointed out that AIN NTs could be used for monitoring ammonia and nitrogen dioxide [6,7], emphasizing the potential interest of such nanostructures.

Following the pioneering work of Wagner and Ellis [8], most of the published literature refers to catalyst-assisted growth of NWs. However, in the specific case of nitride NWs, catalyst-free growth is the main stream, which has led to the spontaneous growth of GaN $\mathrm{NWs}$ on virtually any kind of substrates, including $\mathrm{Al}_{2} \mathrm{O}_{3}$ [9], $\mathrm{Si}[10-12], \mathrm{SiO}_{2}[13,14], \mathrm{TiN}[15]$, graphene [16,17] diamond [18] and more. In addition, the more recent development of selective area growth (SAG) has opened a new route to the growth of NWs using masked substrates. Eventually, the formation of top-down NWs by selective evaporation of a masked GaN layer was demonstrated by Sergent and co-workers [19], emphasizing the versatility of catalyst-free NW growth/elaboration processes.

The nucleation mechanism of such catalyst-free GaN NWs is a complex process, which involves chemical interaction between $\mathrm{Ga}, \mathrm{N}$ and the underlying substrate [20-22]. A detailed analysis of the nucleation mechanism is out of the scope of this article which aims at discussing the elongation mechanism of the NWs in steady-state regime, far from the 
nucleation stage. A special attention will be paid to the role of metal adatom diffusion along the sidewalls as well as on the top surface. The particular case of an adatom diffusion length smaller or comparable to the upper diameter of NWs will be discussed in detail. It will be shown that the spontaneous superlattice formation in ternary III-nitride NWs as well as the formation of AIN nanotubes have a common origin, related to the metal diffusion rate along the sidewalls and the existence of diffusion barrier from sidewalls to top and from top to sidewalls.

\section{Growth of spontaneously nucleated GaN NWs}

Since the pioneering work of Yoshizawa et al. [9] and Sanchez-Garcia et al. [10], III-N nanowires have become a field of considerable interest, related to the remarkable structural and optical properties of these nanostructures and to their potential applications for optoelectronic devices. In the recent years, numerous reports on the plasma-assisted molecular beam epitaxy (PAMBE) growth of self-nucleated GaN NWs have been published. In the steady state regime, i.e. following the nucleation step, the elongation of the GaN NWs is governed by Ga adatom diffusion from the basal plane to the NW and then along their side walls up to the NW top, following the model proposed by Debnath et al. [11]. Consequently, the Ga flux, $\Phi_{\mathrm{Ga}}$, consists of two contributions, namely $\Phi_{\mathrm{Ga}}$,nom and $\Phi_{\mathrm{Ga}}$,diff, where $\Phi_{\mathrm{Ga} \text {,nom }}$ is the nominal Ga flux impinging on top of the NWs and $\Phi_{G a}$, diff the Ga flux impinging on the base plane and/or the NW side walls and diffusing along them to the top. It has to be pointed out that the absolute value of $\Phi_{G a}$ is difficult to quantify, due to the temperaturedependent Ga desorption rate from both top and side walls. In addition, it appears that $\Phi_{G a}$,diff depends on the Ga diffusion rate on the m-plane GaN side walls as well as on the total Ga flux reaching them, which may be affected by a shadow effect related to NW density.

The evidence that the GaN NW growth is governed by $\Phi_{\mathrm{Ga}}=\Phi_{\mathrm{Ga}, \text { nom }}+\Phi_{\mathrm{Ga}}$ diff is provided by the comparison of the growth rate of NWs with respect to the growth rate of layers as a function of $\Phi_{\mathrm{Ga}}$,nom. In $\mathrm{N}$-rich regime (or Ga-limited), a higher growth rate is systematically observed for the NWs, assigned to diffusion along the side walls [23], till reaching the Ga-rich (or N-limited) regime. At the onset of the Ga-rich regime, the growth 
rate of both NWs and layers is converging toward a common value prior to stabilizing at a saturation value limited by the $\mathrm{N}$ flux.

In the vapour-liquid-solid (VLS) model the nucleation of an additional layer on top of a NW is governed by the chemical composition of the metallic catalyst droplet and thermodynamics at the VLS triple line, as described in detail by Glas et al. for GaAs NWs $[24,25]$ and by Gamalski et al. for Ge NWs [26]. By contrast, in the absence of a catalyst, which corresponds to the case of GaN NWs grown by PAMBE, the question then arises to determine where the nucleation of an additional layer is taking place on the top. This issue is expected to basically depend on adatom kinetics on top of the NW, i.e. on the adatom diffusion length with respect to NW diameter as well as on the local adatom density on top. These kinetical parameters are drastically depending on the $\Phi_{\mathrm{Ga}} / \Phi_{\mathrm{N}}$ ratio value and on the details of growth conditions.

Importantly, because of the non-centrosymmetry of the wurtzite structure, the $\{0001\}$ surface exhibits a polar character. GaN polarity is conventionally defined by considering the direction of the Ga-N bond vector parallel to the c-axis, which starts on $\mathrm{Ga}$ and ends on $\mathrm{N}$. If it points towards the surface (respectively the substrate), then the crystal exhibits a Ga-polarity (respectively N-polarity) $[27,28]$. It was found that polarity is significantly affecting the kinetics of adatoms adsorbed on the $\{0001\}$ surface of GaN. First examining the case of Ga-polar GaN layers grown by PA-MBE, two regimes are expected, depending on the $\Phi_{\mathrm{Ga}} / \Phi_{\mathrm{N}}$ value. As theoretically calculated by Zywietz et al., for $\Phi_{\mathrm{Ga}} / \Phi_{\mathrm{N}}>$ 1 the surface diffusion barrier for Ga is low, associated with a large diffusion length [29]. Such a regime corresponds to the so-called Ga-rich (or N-limited) growth conditions and results in smooth surface. Layer-by-layer growth mode is observed in such conditions, eventually associated with a step flow regime at high enough growth temperature, namely at a growth temperature such that the Ga diffusion length is larger than the terrace width [30]. By contrast, for $\Phi_{G a} / \Phi_{N}<1$, i.e. for N-rich (or Ga-limited) growth conditions, the high diffusion barrier for Ga adatoms drastically limits their mobility on the surface, leading to the formation of hillocks and a rough surface. For a high enough $\Phi_{G a} / \Phi_{N}$ value, a peculiar regime is observed characterized by the formation of a self-regulated Ga bilayer on top of the growing (0001) GaN surface, opening a new diffusion channel for sub-surface N diffusion [31]. In general, combined to kinetical parameters and whatever the $\Phi_{G a} / \Phi_{N}$ value, surface 
defects such as steps, grain boundaries, threading edge and screw dislocations are determining to a large extent the growth mode of GaN layers and their morphology.

Similarly, on N-polar (000-1) GaN surface, the Ga adatom diffusion barrier was found to be significantly lower in Ga-rich conditions than in N-rich conditions. Contrary to the case of (0001) GaN, no self-regulated Ga bilayer regime was observed on (000-1). However, for high enough Ga flux, an equilibrium regime corresponding to one monolayer Ga coverage on the surface was put in evidence, followed by Ga accumulation for higher flux [32].

Considering the case of nanocrystals, their morphology is expected to depend on the different surface energies of the different surfaces. The equilibrium shape of GaN wurtzite crystals was calculated by Hong Li et al. [33], predicting a pyramidal shape of Ga-polar crystal termination and a flat surface on N-polar side. Consistently, it was found that GaN NWs grown by selective area growth on Ga-polar GaN template exhibit a pyramidal extremity [34] while N-polar spontaneously nucleated GaN NWs exhibit a flat top [35]. However, these theoretical predictions, based on Wulff constructions and the energy hierarchy of different possible surfaces, are neglecting the kinetical parameters. In particular the surface energy modifications induced by the presence of a Ga layer on the surface combined to the lowering of the Ga adatom diffusion barrier may lead to the stabilization of a flat (0001) surface on top of Ga-polar NWs [36]. In the extreme case of a Ga flux value high enough to result in the formation of a Ga adlayer on top of the NWs, the increased diffusion length of active $\mathrm{N}$ adatoms and their concomitant increased recombination into $\mathrm{N}_{2}$ followed by desorption, considerably decreases the growth rate along the vertical axis. In addition, specific to NW geometry in the case of a Ga flux higher than the N flux, Ga adatoms accumulate in the upper part of the side walls at the frontier with the top due to the limited Ga-consumption rate on top, associated with the $\mathrm{N}$-limited regime. This leads to an increasing GaN nucleation probability on the sides and a lateral widening of the NW top, promoting the formation of inverted hexagonal pyramids [36].

More generally, the influence of the $\Phi_{\mathrm{Ga}} / \Phi_{\mathrm{N}}$ value on the morphology of N-polar GaN NWs was studied in detail by Fernandez-Garrido et al. Starting from the steady state regime, it was demonstrated that an increase of the Ga flux led to a widening of the GaN NWs till reaching a new equilibrium state associated with a larger diameter [37]. As a whole, 
it was demonstrated that the NW diameter was self-regulated so that the effective $\Phi_{\mathrm{Ga}} / \Phi_{\mathrm{N}}$ value on top corresponds to near-stoichiometric conditions [37]. Such a result, namely the widening of the NW top as soon as the effective metal flux is set at a value exceeding $\Phi_{\mathrm{N}}$, was thoroughly investigated in the case of GaN and InGaN NWs. In both cases, growth in metal-rich conditions eventually leads to the spontaneous formation of GaN and InGaN nano-platelets (also called nano-parasols or nano-umbrellas), assigned to a drastic decrease of the vertical growth rate combined with enhanced NW widening [38-41].

Contrary to their GaN layer counterparts, GaN NWs are free of extrinsic nucleation centers such as dislocations and grain boundaries [42], making nontrivial the identification of the nucleation site of an additional layer on top of a growing NW. Indeed, due to the reduced diameter of NWs, conditions can be met for which the Ga adatom diffusion length may be tuned to be larger or smaller than the NW diameter, leading to a nucleation regime of additional layers on top directly affected by the edge [36]. As it will be shown later, in these conditions, the surface morphology of GaN NWs can be engineered by tuning the $\Phi_{\mathrm{Ga}}$ / $\Phi_{\mathrm{N}}$ value.

In order to evaluate the effect of Ga adatom kinetics on the elongation mechanism, Ga-polar GaN NWs (about $500 \mathrm{~nm}$ in diameter) grown by selective area growth (SAG) on a GaN/sapphire wafer were used. On this NW template, a section of GaN was further grown by PAMBE in Ga-rich conditions during 30 minutes. An atomic force microscopy (AFM) picture of these NWs is shown in figure 1, where Ga droplets can be seen on top of each individual NW, as a result of Ga accumulation inherent to Ga-rich growth conditions. In absence of structural defects such as dislocations or grain boundaries on the NW top, this droplet formation is assumed to obey an intrinsic nucleation mechanism. Remarkably, as shown in figure 1a, only one Ga droplet per NW is observed, assigned to a single nucleation event. This suggests that Ga diffusion length in these growth conditions is longer than the NW diameter ( $400 \mathrm{~nm}$ ), so that all Ga atoms on top are collected by the single nucleation center. The relevant parameters of Ga droplet distribution over the top of GaN NWs, namely their diameter, $d$, and height, $h$, and the position of their center with respect to the edge, $e$, along the NW diameter, D, are defined in figure $1 \mathrm{~b}-\mathrm{d}$. The statistical analysis of these parameters for $155 \mathrm{NWs}$ is shown in figure 2. It appears that the Ga droplet radius (d/2) normalized to $D$ (figure $2 a$ ) is markedly peaked at 0.16 . This sharp distribution confirms the 
average homogeneity of the total Ga flux on top of the NWs, which is a combination of the Ga flux directly impinging on top and of the Ga flux diffusing from the side walls towards the top.
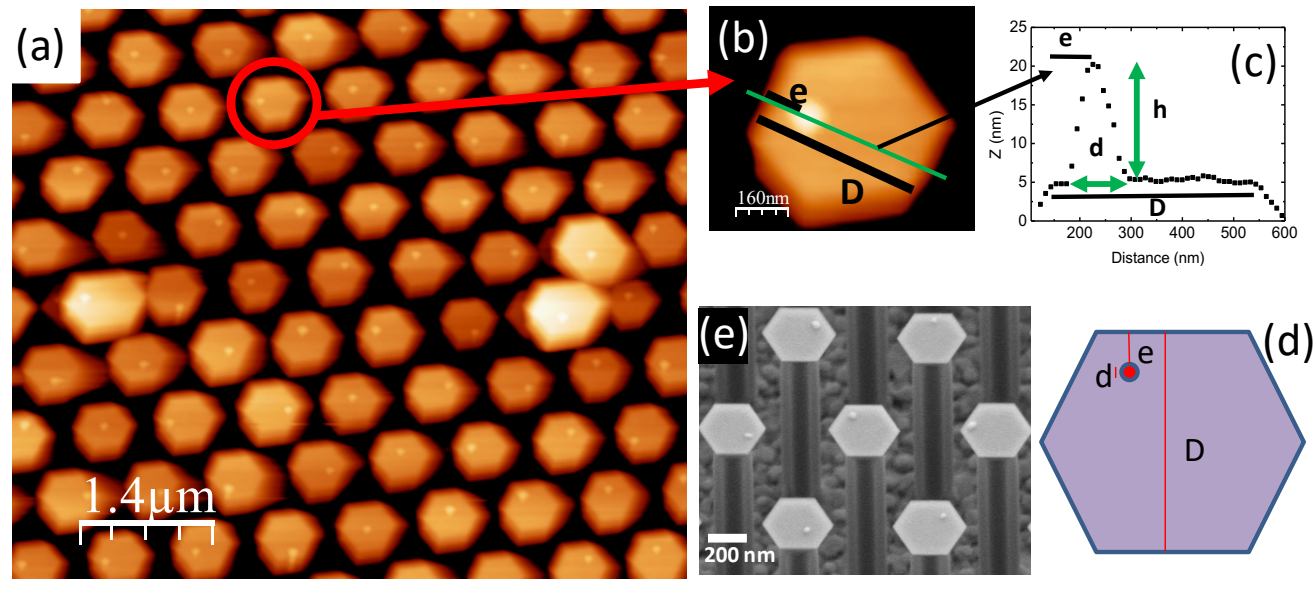

Figure 1 : (a) AFM image of GaN NWs with Ga droplets on top. (b) Enlargement of a single NW with a Ga droplet on top. (c) Morphology profile along the green line shown in (b), allowing one to determine the $e, d, h$ and $D$ parameters defined in (d). (e) Scanning electron microscopy image showing the enlarged morphology of the GaN NWs on top of the GaN stem substrate.

As shown in figure $2 b$, the Ga droplet location on top of the NWs is widely dispersed. However, it clearly appears that the nucleation probability for an e/D ratio value lower than 0.15 rapidly decreases. This is a clue that the diffusion barrier from top to NW walls effectively decreases the Ga presence probability close to the edges and concomitantly the droplet nucleation probability. For e/D larger than 0.15 , the continuous decrease of the droplet distribution from the edge to the center most likely reflects the Ga adatom density on top and the associated droplet nucleation probability. The variation of the Ga drop volume as a function of $D$ is plotted in figure $2 c$. Actually, the progressive widening of the GaN NW in Ga-rich conditions (see figure 1e) is expected to result in the formation of an additional diffusion barrier at the frontier between the GaN stem and the enlarged section. Accordingly, in these specific growth conditions, the Ga flux diffusing from the side walls, $\Phi_{\mathrm{Ga}}$,diff, can be considered as negligible. Consistently with this statement, the droplet volume as a function of $D$ is expected to be mostly proportional to the upper surface assuming a $G a$ 
sticking coefficient independent of diameter. In agreement with this hypothesis, the droplet volume is proportional to $D^{2}$ (shown in figure $2 c$ ), consistent with the dominant influence of the top surface associated with a relatively negligible importance of the sidewalls. The equivalent Ga coverage was found to be $0.42 \pm 0.15 \mathrm{~nm}$, consistent with the $0.49 \mathrm{~nm}$ value expected for the thickness of the Ga bilayer, which is present on (0001) GaN surface grown in Ga-rich conditions at high temperature [31]. This suggests that the Ga droplet nucleation might occur during the cooling process at the end of the growth, when crossing the stability line of the Ga bilayer regime as a function of temperature [30].
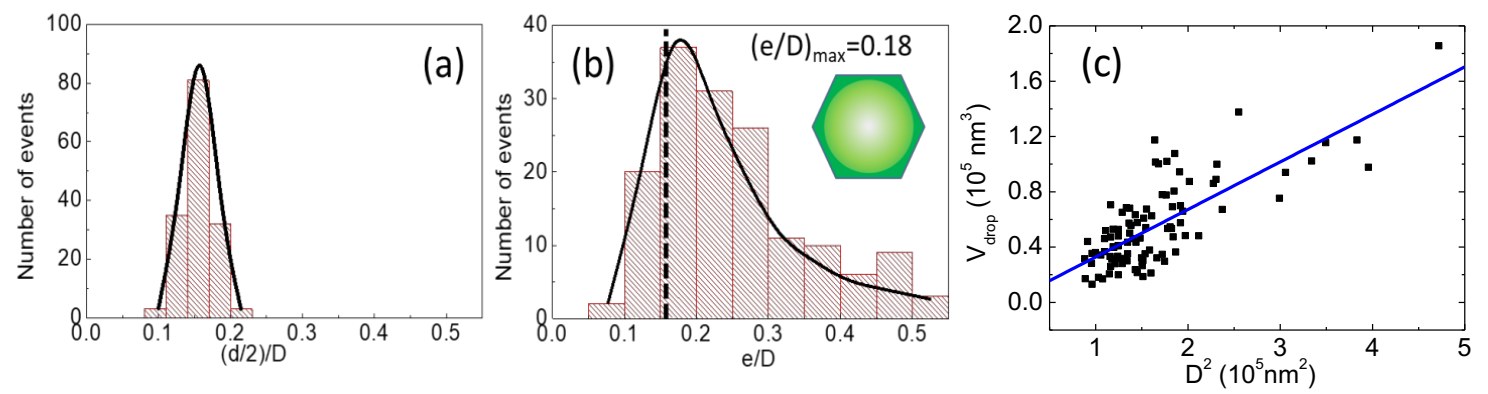

Figure 2: (a) Statistical Ga droplet distribution. (b) Statistical distribution of the Ga droplets on the surface, with indication of the mean radius (dashed line). Full lines are guides to the eyes. Parameters $d, D$, and e are defined in figure 1 (d). (c) Drop volume as a function of $D^{2}$, exhibiting a linear dependence.

If now considering the case of GaN NWs grown with a nominal $\Phi_{G a} / \Phi_{N}$ value equal to 1.6, no Ga droplets are present on top, as shown in figure 3a. These moderately Ga-rich conditions correspond to a long Ga adatom diffusion length on the top (0001) surface, without Ga accumulation. The height profile across the diameter is shown in figure $3 \mathrm{~b}$ and reveals a smooth concave shape, which we assign to a higher nucleation probability in the periphery [36]. This process is schematized in figure 3c, where the combined effect of the diffusion barrier from top to walls and of the additional Ga flux diffusing from the side walls to the top is expected to result in a radial Ga gradient decreasing from the side to the center.

By contrast, when GaN NWs growth is performed using a nominal $\Phi_{G a} / \Phi_{N}$ value equal to 0.35 , their top surface is rough, as shown in figure 4 a. This feature is further put in evidence by the height profile in figure $4 \mathrm{~b}$. Such a roughness is assigned to multiple 
nucleation events associated with a short Ga adatom diffusion length in N-rich growth conditions lying in the $30-50 \mathrm{~nm}$ range $[36,43]$. However, the AFM data in figure 4 also reveal an accumulation of material in the periphery, assigned to the diffusion of Ga along the sidewalls till the top surface. As schematized in figure $4 c$ and $4 d$, due to the short Ga adatom diffusion length on top, the extra Ga flux coming from the side walls is responsible for the peripheral bulging of the GaN NW top surface.

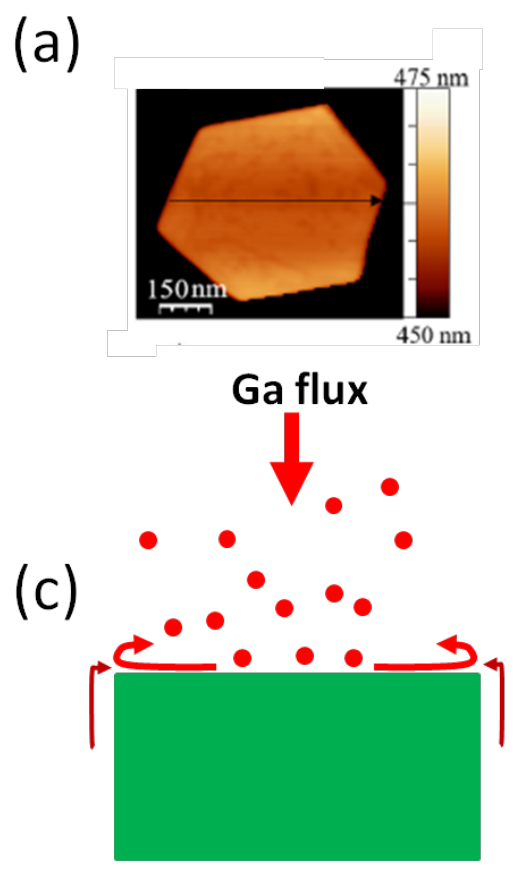

(b)

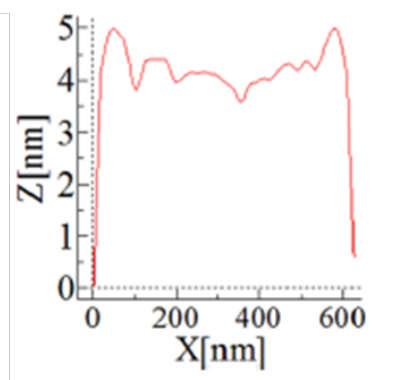

(d)

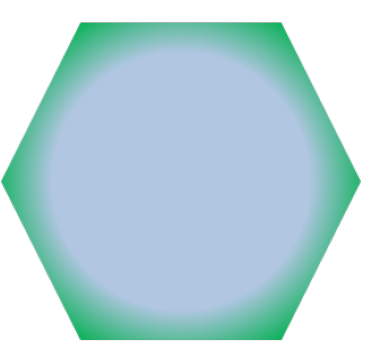

Figure 3 : (a) AFM image of a GaN NW grown with a nominal $\Phi_{G a} / \Phi_{N}$ value equal to 1.6. (b) Height profile along the black line in (a), showing the concave shape assigned to a Ga excess in its periphery. (c) Schematics of exposure of NW surface to both an impinging Ga flux and a Ga flux diffusing from the side walls, with the effect of a peripheral diffusion barrier leading to (d) a Ga presence probability higher (green) in the periphery of GaN NWs than in their center (blue). (Adapted with permission from Nano Lett. 2019, 19, 4250-4256. Copyright 2019 American Chemical Society). 

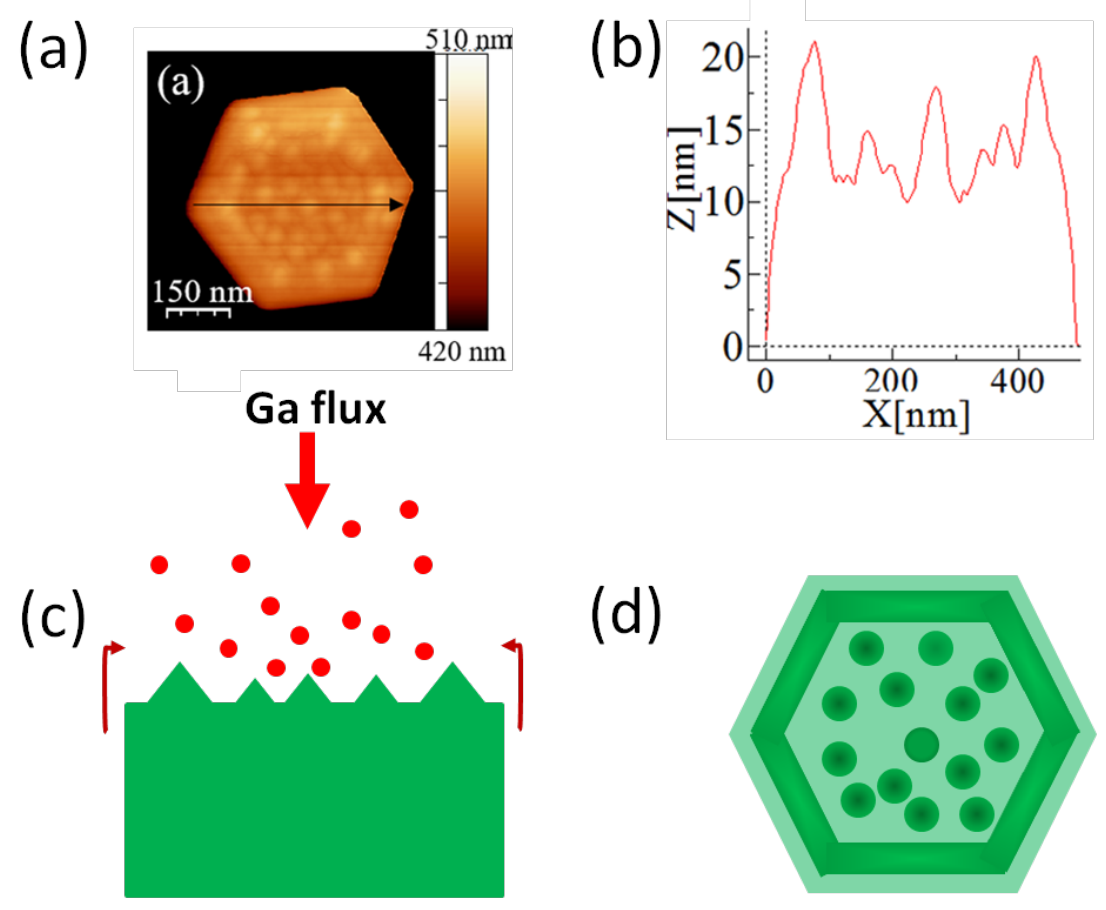

Figure 4: (a) AFM image of a GaN NW grown with a nominal $\Phi_{G a} / \Phi_{N}$ value equal to 0.35. (b) Height profile along the black line in (a), showing the rough top surface associated with an enhanced nucleation rate in the periphery. (c) Schematics of exposure of NW surface to both an impinging Ga flux and a Ga flux diffusing from the side walls, in conditions of short Ga adatom diffusion length on the top leading to $(d)$ a rough surface and enhanced nucleation rate in the periphery of GaN NWs. (Adapted with permission from Nano Lett. 2019, 19, 4250-4256. Copyright 2019 American Chemical Society).

From the data above, it is concluded that the Ga adatom diffusion length on the top of GaN NWs varies from 30-50 nm in N-rich growth conditions till several hundreds of $\mathrm{nm}$ in Ga-rich conditions. As a consequence, it is speculated that in the case of spontaneously formed GaN NWs, with a diameter in the 30-100 nm range, a single nucleation event is triggering the formation of each additional layer. Due to the presence of a diffusion barrier at the edge between top and side walls, it is furthermore speculated that the nucleation event is not occurring at the edge, as proposed by Fernández-Garrido et al. [37], but close to the edge, at a distance corresponding to the maximum probability of presence of $\mathrm{Ga}$ adatoms diffusing on the top and repelled from the edge due to the presence of the barrier. While this effect is hardly noticeable in the case of NWs exhibiting a small diameter, it 
cannot be neglected in the case of larger NWs such as those shown in figure 4 and leads to a marked bulging ring on the NW top surface.

Finally, it should be pointed out that the polarity is not expected to basically change the phenomenology governing the NW morphology dependence on the $\Phi_{\mathrm{Ga}} / \Phi_{\mathrm{N}}$ ratio value. As a matter of fact, neither the diffusion along the non polar side walls nor the accumulation on top of the side walls responsible for NW enlargement in Ga-rich conditions, are affected by the NW polarity. By contrast, the height of the diffusion barrier from side walls to top and vice-versa is likely to depend on the polarity. However, for both polarities, the Ga adatom diffusion length is significantly larger in Ga-rich conditions than in N-rich ones, providing the driving force controlling the top morphology of NWs.

III. Spontaneous superlattice formation in InGaN and AIGaN nanowires

Spontaneous chemical ordering was observed at the scale of several tens of nanometers in N-polar AlGaN NWs [44], leading to the spontaneous formation of alternated Ga-rich and Al-rich sections. Similar effects had been observed in $\mathrm{CdS} / \mathrm{Ag}_{2} \mathrm{~S} \mathrm{NWs}$, which were assigned to the spontaneous conversion of the initial NW of single chemical composition into a stripped pattern of alternated $\mathrm{CdS}$ and $\mathrm{Ag}_{2} \mathrm{~S}$ sections [45]. Based on theoretical calculations, the driving force for this evolution was identified as a kinetically-driven minimization of the stored elastic energy, shedding light upon the importance of cationexchange reactions in NWs. While the large binding energy of both GaN and AIN makes unlikely such a cation exchange in the core of AIGaN NWs, the situation is not the same at the surface. Indeed, vertical exchange of Ga and Al was put in evidence when capping GaN quantum dots or quantum wells with AIN, which was assigned to the high binding energy of AIN compared to GaN. It was furthermore shown that the cation exchange mechanism was thermally activated and dependent on the strain state of the subjacent GaN nanostructure [46]. The reduced diameter of NWs and their eased elastic strain relaxation favoured by the large amount of free surface suggest that the alternate formation of Ga-rich and Al-rich sections, several nanometers thick, could be assisted by a Ga/Al vertical exchange mechanism, depending on the local Ga/Al adatom density on the NW top. Along these lines, the formation of a pure AIN section on top of GaN NWs exposed to both a Ga and an Al flux, 
which is unfavourable in terms of stored elastic energy, could result from the initial Ga/Al exchange leading to the formation of a pure AIN layer, next followed by preferential nucleation of AIN on the first AIN layer [44]. In addition, besides chemical segregation at the scale of some nanometers, spontaneous ordering was commonly observed at the scale of single atomic layers in the case of AIGaN [47-49], and InGaN [41] NWs. More sporadically, the observation of phase separation and spontaneous superlattice formation (SSF) was reported for InGaN [50], AIGaN [51-55] and AIGaInN [51] layers. Such superlattices consist of an alternate and periodic stacking of GaN and $\operatorname{lnN}$ or AIN layers. Several types of stacking sequences were observed, namely $1: 1,3: 1,10: 2$ depending on , the growth temperature and the relative ratio of the metallic fluxes involved (In/Ga and Al/Ga for InGaN and AlGaN, respectively) [52]. Furthermore, it was demonstrated by Pakula et al. [55] that the propensity to form spontaneous superlattices also depends on the state of the growing surface, prior to the deposition of the AIGaN layer.

A rigorous explanation for the SSF was first given by Northrup et al. to account for their occurrence in the case of InGaN [56]. These authors have shown that (10-11) surfaces of wurtzite III-nitrides exhibit two types of adsorption sites, corresponding to one or two bonds formed between the impinging metal atom and the $\mathrm{N}$ atom in the layer. The difference in bond length and strength results in preferential incorporation of the metallic species either in the one or the other of the two sites, leading to the formation of a 1:1 spontaneous superlattice. As pointed out by Lymperakis et al. [57], the atomic structure of the (10-11) surface is identical to the structure of the surface created by the formation of a step of height $c$, which allows one to link SSF to the roughness of the growing surface. The same kind of explanation was invoked by Albrecht et al. to account for SSF in AIGaN. [58], consistent with the experimental results of Pakula et al., which emphasize the role of the steps behind the SSF mechanism [55].

Turning back to the specific case of N-polar AIGaN NWs, a variety of periodicities was observed at the scale of individual NWs, pointing out towards a formation mechanism drastically depending on the nominal Ga/Al flux ratio value and local growth conditions $[44,59]$. As an example, high angle annular dark field (HAADF) high resolution scanning transmission electron microscopy (HRSTEM) images of the two top sections of two different AIGaN NWs corresponding to the same sample are shown in figures $5 \mathrm{a}$ and $5 \mathrm{c}$, obtained with 
a probe corrected FEI Titan Themis microscope working at $300 \mathrm{kV}$. The growth was performed at about $820^{\circ} \mathrm{C}$, using a nominal $\Phi_{\mathrm{Ga}} / \Phi_{\mathrm{Al}}$ flux ratio of $50 \%$. Figures $5 \mathrm{~b}$ and $5 \mathrm{~d}$ show the HAADF intensity profiles along the growth axis of the NW shown in figures $5 a$ and $5 c$, respectively. The periodical variation of the contrast is assigned to periodical fluctuations of AIN/GaN molar fraction, demonstrating a fluctuating spontaneous chemical ordering varying from NW to NW.
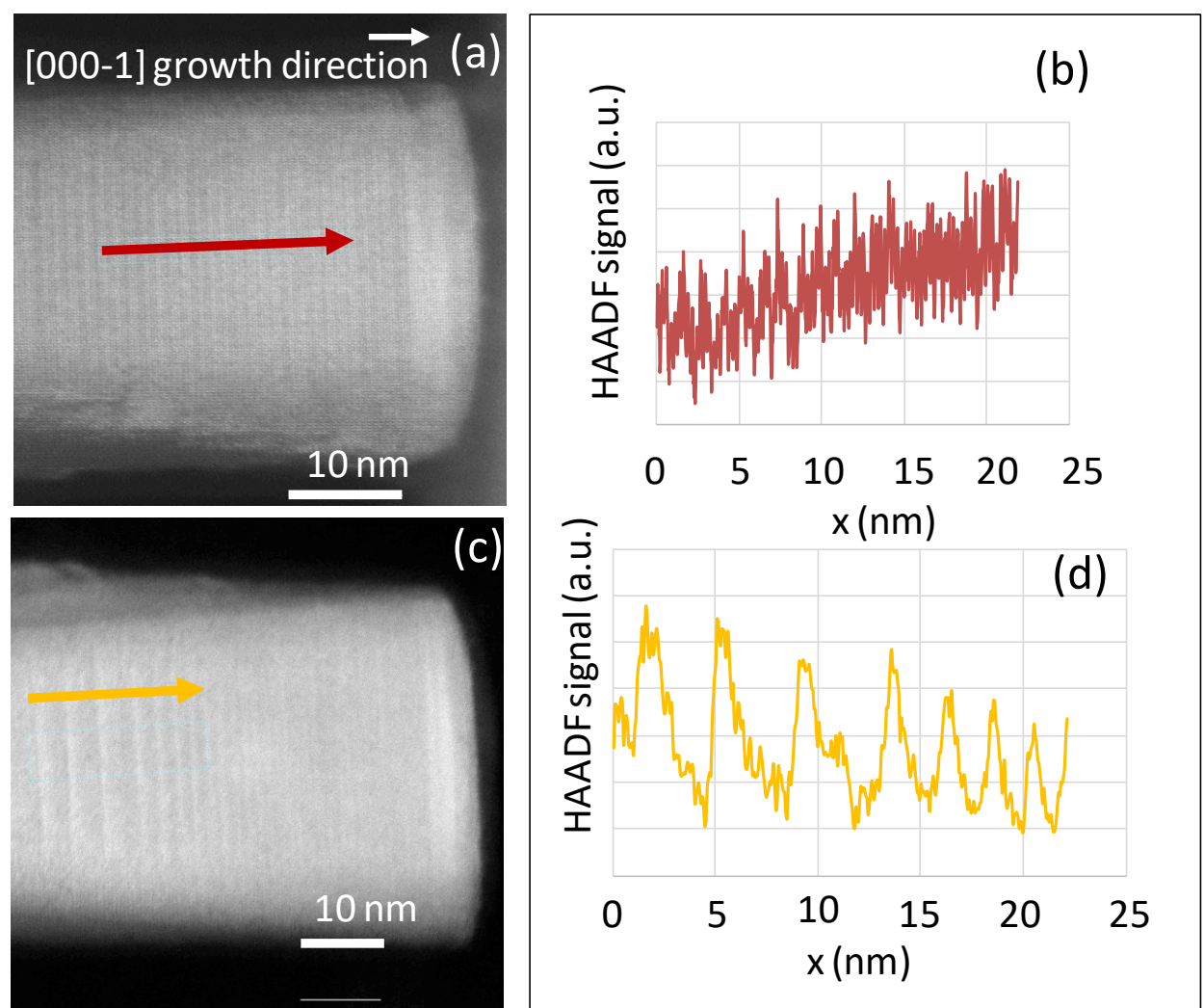

Figure 5: (a,c) HAADF-HRSTEM image of two different AIGaN NWs of the same sample with a nominal Ga/Al flux ratio of 0.5 . (b,d) HAADF intensity profile along the growth axis, qualitatively showing a periodical variation of the local Al/Ga content.

As a further evidence of spontaneous chemical ordering in AIGaN NWs, figure 6 shows energy dispersive Xrays spectroscopy (EDX) analysis of single AIGaN NWs. Growth was performed at $725{ }^{\circ} \mathrm{C}$, the nominal Ga/Al flux ratio was 0.6. HAADF-STEM images and EDX maps of Al K-line (red) and Ga K-line (green) were acquired at $200 \mathrm{kV}$ on a probe corrected 
Titan Themis FEI microscope equipped with four Silicon Drift Detectors using the QUANTAX800 software provided by BRUKER. Acquisition was performed at a beam current of $450 \mathrm{pA}$ with a beam convergence angle of $20 \mathrm{mrad}$ and a pixel size of $0.7 \mathrm{~nm}$. As clearly seen in figure 6, a random alternance of AIN and GaN layers stacked along the growth axis is put in evidence, ultimately consisting of AIN/GaN monolayer stackings mixed with thicker, alternated Al-rich and Ga-rich sections.
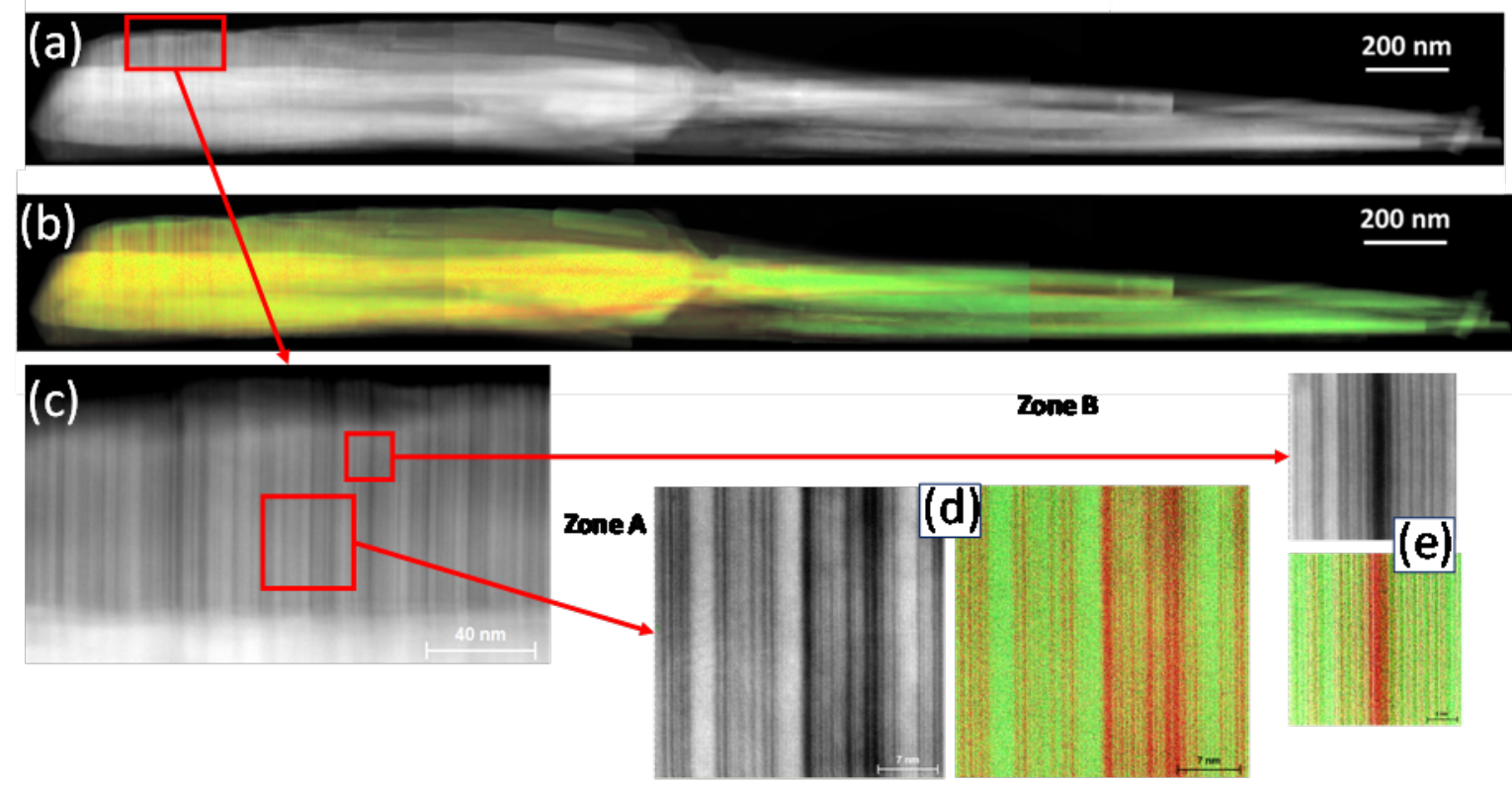

Figure 6: (a) HAADF-STEM images of AIGaN NWs grown on a GaN NW base with (b) the corresponding EDX mapping (Ga in green, $A$ l in red). (c) Enlarged view of the inset in (a) showing the formation of a spontaneous superlattice. (d) Further zooming of the squared zone in (c) showing a local HAADF-STEM image with the corresponding filtered HAADF +EDX map. (e) The same in another region of (c).

As established in section II, the elongation mechanism of GaN NWs is governed by the diffusion of Ga adatoms along the side walls toward the top surface, which results in material accumulation in the periphery of the top surface of (0001) GaN NWs [36]. Whatever the physical mechanism in action, i.e. either governed by small or large Ga adatom diffusion length, the resulting concave shape of the (0001) NW top surface and the associated 
peripheral bulging lead to the formation of steps, as schematized in figure 8-b. The formation of such steps in the periphery of the NW top surface is expected to be the driving force for SSF commonly observed in AIGaN NWs, as shown in figures 5 and 6.

The similar spontaneous formation of superlattices in InGaN NWs is illustrated in figure 7. Experiments were performed using a $300 \mathrm{kV}$ TITAN Ultimate miscroscope in HAADFSTEM mode. The convergence angle was $20 \mathrm{mrad}$. The image contrast is essentially sensitive to $Z^{\alpha}$ where $\alpha$ is about 2 [60]. HAADF-STEM image of the head of an InGaN NW grown on a GaN NW base is displayed in figure 7a, putting in evidence a Z-contrast which can be used to locate In-rich layers, shown in figure $7 \mathrm{~b}$ and inset.
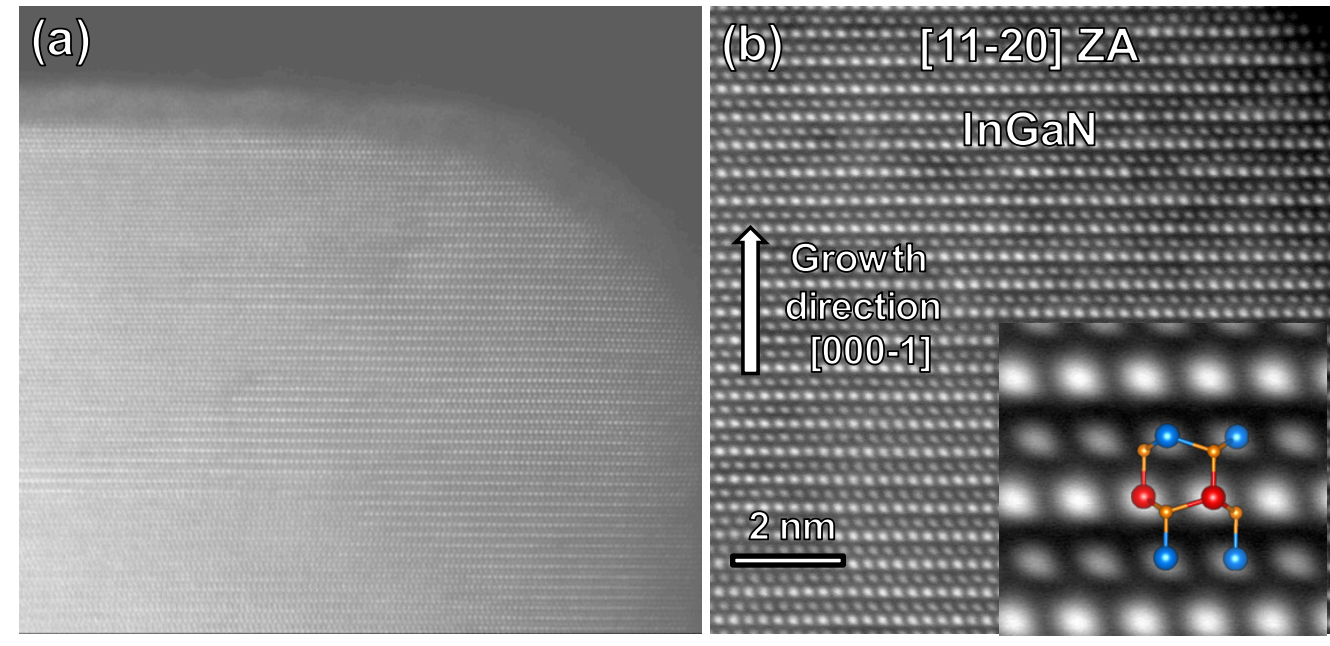

Figure 7: (a) HAADF-STEM images of the head of an InGaN NW grown on a GaN NW base showing a Z-contrast image which can be used to locate In-rich layers (b) High resolution zoom of the external part of the NW outlining the spontaneous formation of alternately Inrich and Ga-rich layers, mostly present in the external part of the NW. Inset: atomistic model with In (red) and Ga (blue) atoms alternating in successive (000-1) planes.

Importantly, in the case of ternary NWs, the diffusivity of both atomic species is significantly different. Thus, the relatively short diffusion length of Ga with respect to In combined to the moderate growth temperature required by the limited temperature stability of InGaN alloys leads to the formation of a GaN shell around InGaN NWs [61,62]. Similarly, an AIN shell is observed around AIGaN NWs, as a result of the smaller diffusion length of Al compared to $\mathrm{Ga}$ [44]. Besides the increasing adatom density of the less-diffusing 
metal species on the side walls and the kinetically-driven formation of a shell, it is expected that the peripheral bulging will be enriched in the less mobile metal species.

On this experimental basis, the possible InGaN and AIGaN SSF mechanism in NWs is schematized in figure 8. The consequence of the kinetical processes described in section II, i.e. the formation of a peripheral bulging when growing GaN NWs is illustrated in figure 8a. Importantly, it should be remarked that the existence/height of this bulging directly depends on the Ga adatom diffusion length with respect to NW diameter, so that it can be absent in case of NWs exhibiting a diameter smaller than the minimum Ga diffusion length of 30-50 $\mathrm{nm}$ in $\mathrm{N}$-rich growth conditions. When switching to the growth of an InGaN section, the facets of the peripheral bulging act as seeds for the spontaneous formation of a GaN/InN superlattice, as illustrated in figures $8 \mathrm{a}$ and $8 \mathrm{~b}$. Simultaneously, the increased nucleation probability of the less mobile metal species, namely $\mathrm{Ga}$, on the side walls top promotes the spontaneous formation of a GaN shell around the InGaN core. Similarly, in the case of AIGaN, the facetted bulging at the top of the GaN NW section induces the formation of an AIN/GaN superlattice surrounded by a spontaneously formed AIN shell as shown in figure 8c.

As a conclusion of this section, it appears that the unique character of metal adatom kinetics driving the growth of NWs leads to a unique morphology of the top, depending on growth conditions such as the temperature and the chemical nature of atomic species involved. This combination is consistent with the frequent observation of SSF in ternary III-N NWs. Finally, it is worth stressing that high resolution electron microscopy observations are usually limited to a reduced number of NWs for technical reasons. However, extended diffraction anomalous fine structure (EDAFS) experiments recently reported by Belloeil et al. have demonstrated a systematic Ga clustering at the scale of next nearest neighbours [63]. These results, consistent with the alternate formation of Ga-rich/Al-rich sections, suggest that spontaneous chemical ordering in AlGaN NWs is indeed the general case, further emphasizing the role of kinetics in NWs. In addition, these structural features are also consistent with optical spectroscopy experiments, which have led to the systematic observation of carrier localization in ensembles of AIGaN NWs [44], and have provided evidence of quantum dot-like localization centers in single NWs [64]. 
(a)

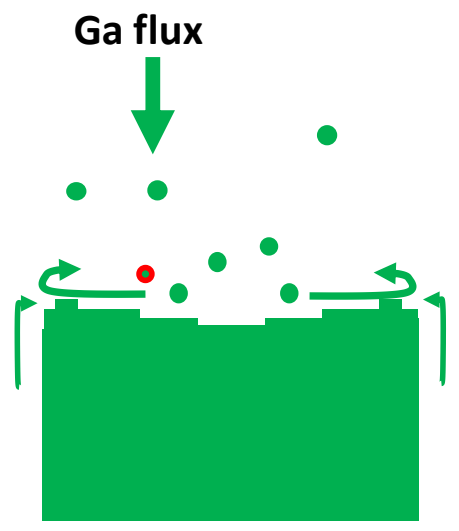

(b)

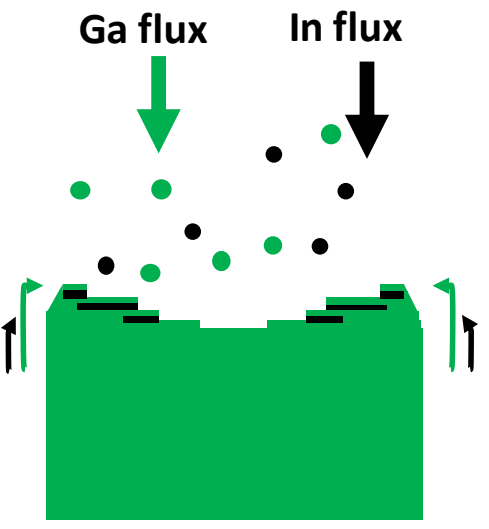

(c)

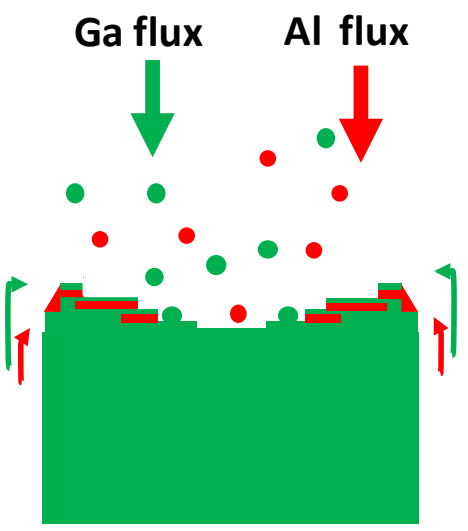

Figure 8: (a) Schematics of metal atom fluxes impinging on both NW top and sidewalls and acting in their elongation process. (b) The preferential nucleation in the periphery is resulting from metal adatom kinetics and leads to the formation of spontaneous InGaN superlattices and of a GaN shell. (c) The same for AlGaN, with formation of an AIN shell.

\section{Growth of AIN nanotubes}

The growth of GaN NTs by chemical vapour deposition (CVD) [65], molecular beam epitaxy (MBE) [66,67] and halide vapour phase epitaxy (HVPE) [68] techniques were reported. In their pioneering work, Goldberger et al. have used a ZnO NW template for depositing a GaN shell around it, followed by removal of the $\mathrm{ZnO}$ core by thermal treatment [65]. A similar method was recently used by Durand et al. to grow thin wall NTs consisting of InGaN/GaN multi-quantum wells around a sacrificial GaN core [69]. By contrast, reports on the growth of AIN NTs are scarce [70]. In this section, we report the Mg-assisted growth of such AIN NTs by PAMBE on top of AIN NW template. Consistent with the above considerations, the formation of the NTs is assigned to peripheral nucleation of AIN on top of AIN NWs, which is triggered by the enhanced diffusion of Al adatoms along the AIN NW sidewalls, due to the surfactant effect of Mg.

The N-polar AIN NWs and NTs were elaborated by catalyst-free PAMBE under nitrogen rich conditions. The substrate used for the growth of the structures was Si (111). Following degreasing and dipping in HF, the substrate was mounted In-free on a molybdenum sample holder and introduced in the MBE growth chamber. Next the Si substrate was outgassed during 30 minutes at $250^{\circ} \mathrm{C}$ and further heated up to the NT growth 
temperature, which was varied between 835 and $930{ }^{\circ} \mathrm{C}$. It has to be pointed out that the direct growth of AIN NWs on Si is challenging. A high temperature is required, in the 900$1000{ }^{\circ} \mathrm{C}$ range, to compensate for the low diffusion lengths of $\mathrm{Al}$ adatoms on both NW top and side walls, compared to Ga [71]. To overcome this difficulty, N-polar GaN NWs were first grown to be used as a template for further AIN NW/NT growth.

The length of these GaN NW stems was approximately $1 \mu \mathrm{m}$. Next $\mathrm{n}$-type and p-type AIN sections, about 500 to $800 \mathrm{~nm}$ each, were grown on top of the GaN stems. The diameter of the AIN NW/NT sections along the growth direction and from sample to sample typically varied from $70 \mathrm{~nm}$ up to $300 \mathrm{~nm}$. For all samples, the n-type doping of the lower AIN lower section was achieved using the same experimental conditions, namely a Si cell temperature of $900{ }^{\circ} \mathrm{C}$. The growth conditions of the five different samples under investigation are summarized in Table 1. The resulting GaN/AIN NW/NT heterostructures were characterized by field-emission scanning electron microscopy (FESEM) imaging using a ZEISS Ultra 55 microscope. As shown in figure 9, the formation of AIN NTs was found to be associated with Mg-doping and dependent on both the growth temperature and $\mathrm{Mg}$ cell temperature, i.e. on the Mg flux.

\begin{tabular}{|c|c|c|c|c|c|}
\hline 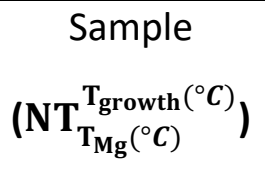 & $\mathbf{N T}_{220}^{835}$ & $\mathrm{NT}_{220}^{900}$ & $\mathbf{N T}_{220}^{930}$ & $\mathrm{NT}_{\mathrm{No} \mathrm{Mg}}^{930}$ & $\mathrm{NT}_{250}^{930}$ \\
\hline $\mathrm{T}_{\text {growth }}\left({ }^{\circ} \mathrm{C}\right)$ & 835 & 900 & 930 & 930 & 930 \\
\hline $\mathrm{T}_{\mathrm{Mg}}\left({ }^{\circ} \mathrm{C}\right)$ & 220 & 220 & 220 & - & 250 \\
\hline
\end{tabular}

Table 1 : Growth conditions of the different AIN NTs samples

For a given growth temperature of $930{ }^{\circ} \mathrm{C}$ (figure $9 \mathrm{a}, \mathrm{b}, \mathrm{c}$ ), no AIN NTS are observed in absence of additional Mg flux during AIN growth. Furthermore, even when using such an additional and increasingly high Mg flux during the growth of the upper AIN NW section, no NTs are observed, which is assigned to the low sticking coefficient of Mg. Consistently, when using a Mg flux corresponding to a $\mathrm{Mg}$ cell temperature of $220^{\circ} \mathrm{C}$ (figure $9 \mathrm{~d}, \mathrm{e}, \mathrm{f}$ ), AIN NTs are observed for the two lowest growth temperatures of 900 and $835^{\circ} \mathrm{C}$. 

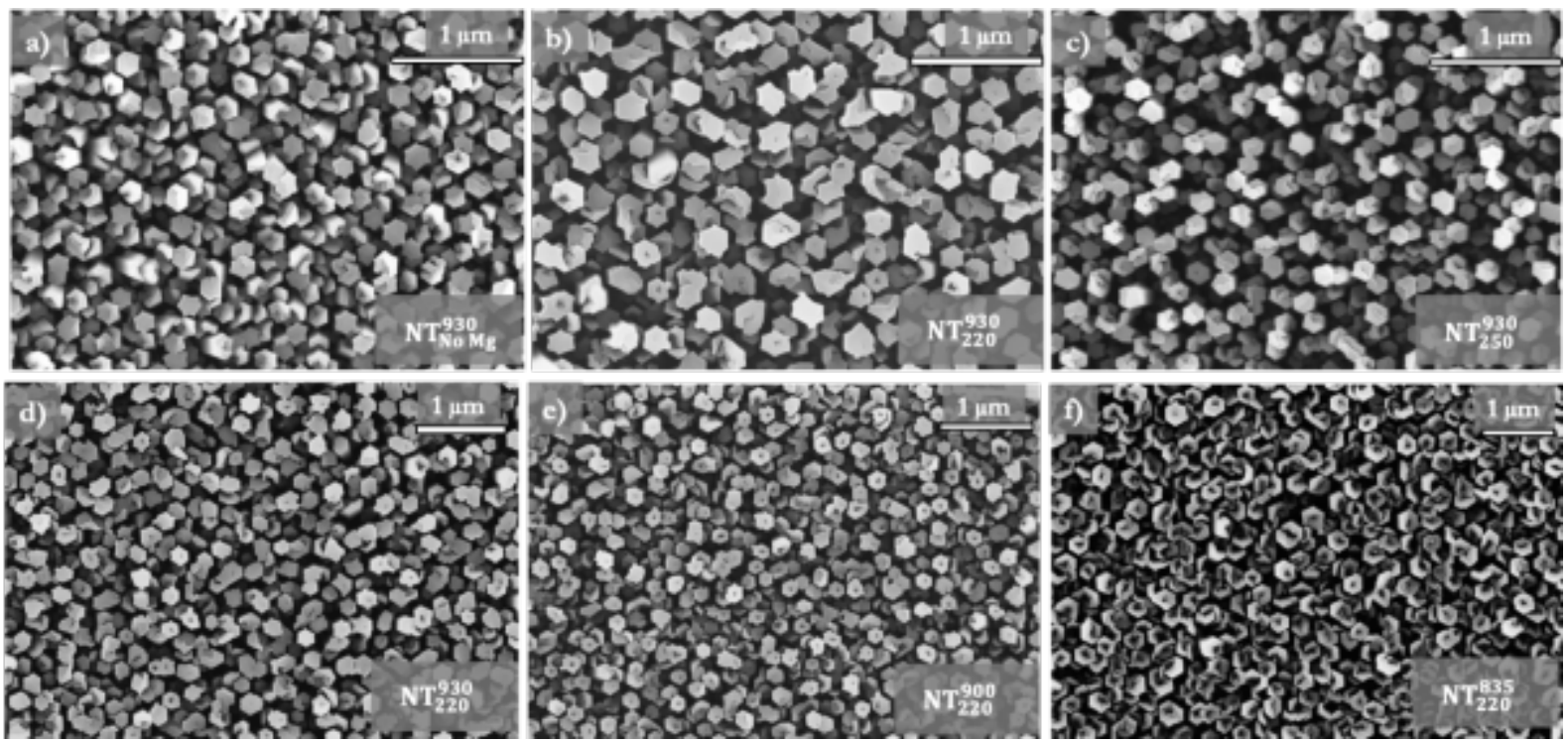

Figure 9: Top-view scanning electron microscopy pictures of the samples under investigation.

(a) Reference sample grown without $\mathrm{Mg}$, (b-f) samples grown according to conditions reported in table 1.

For the sample grown at $835^{\circ} \mathrm{C}$, for a $\mathrm{Mg}$ cell temperature of $220^{\circ} \mathrm{C}\left(\mathrm{NT}_{220}^{835}\right)$, further insight in the morphology of the AIN NTs was obtained by a combination of atomic force microscopy (AFM), high-resolution transmission electron microscopy (HRTEM) and HAADF STEM experiments. As shown in figure 10a, b and c, the NTs depth can exceed $800 \mathrm{~nm}$ and is associated with Mg doping while no NT formation was observed in the Si-doped AIN NW section. Interestingly, the presence of about $1.510^{5} / \mathrm{cm}$ randomly formed stacking faults (SFs) can be seen in figure $10 \mathrm{~d}$ and inset. It is worth pointing out that SF formation was observed in the case of Mg-doped GaN NWs, the density of which was dramatically increasing as a function of Mg flux [72]. This feature suggests that the SF observed in AIN NTS could be related to the exposure to $\mathrm{Mg}$ flux, possibly assigned to the enhanced lateral growth rate on the $\mathrm{m}$-plane side walls in presence of $\mathrm{Mg}$, as discussed later. 

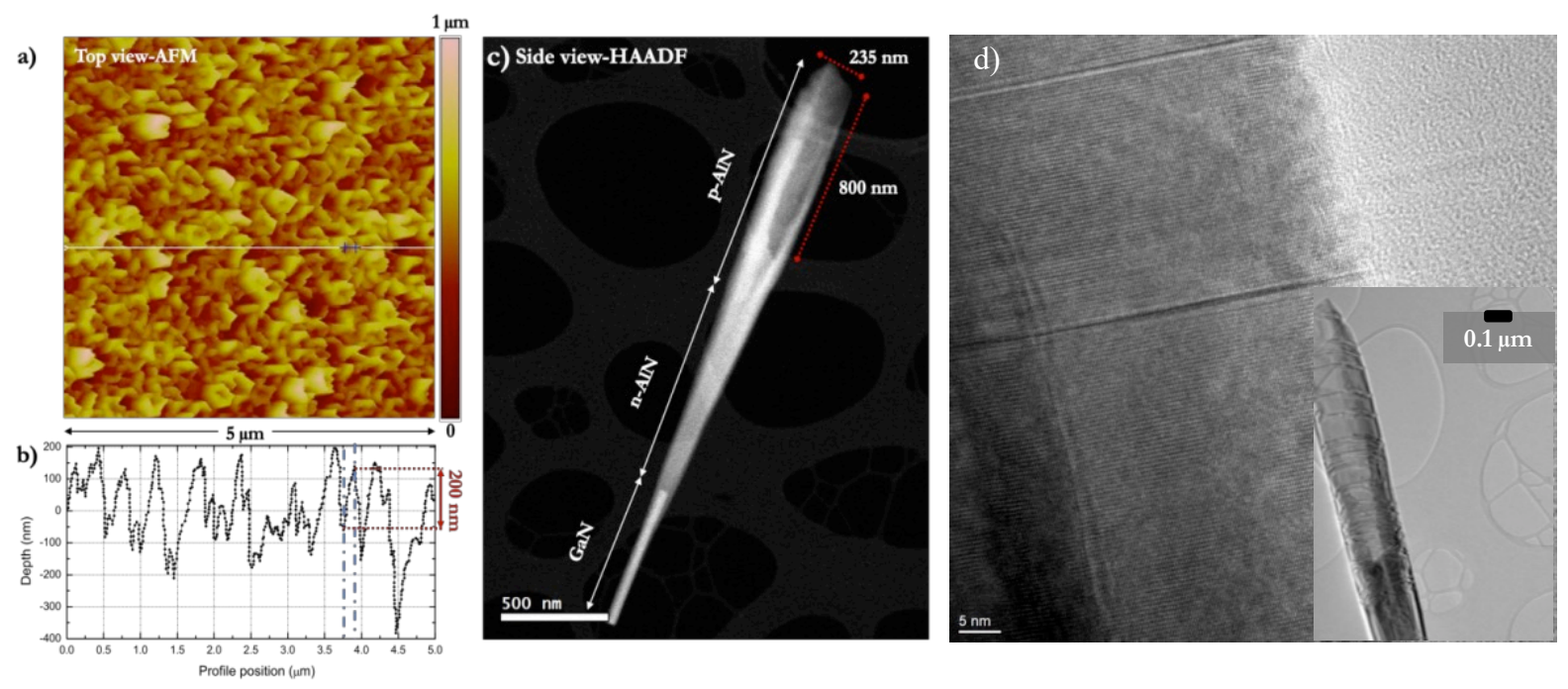

Figure 10: sample $\left(\mathrm{NT}_{220}^{835}\right)$. (a) AFM top view of AIN nanotubes. (b) Depth profile through the line shown in (a). (c) Side view-HAADF view of a single NW/NT. The GaN NW base is shown. The n-AIN NW and AIN NT sections length are shown. (d) High resolution TEM image of an AIN NT side wall, showing the presence of stacking faults. Inset: TEM image of a single dispersed AIN NT. Stacking faults are visible.

As shown in section II, the elongation mechanism of GaN NWs is governed by both the metal flux impinging on the top and the additional flux diffusing from the side walls to the top. As a whole, and whatever the value of the metal/ $\mathrm{N}$ flux ratio, it was concluded that both the reduced nucleation probability on the sidewalls and the eased diffusion toward the top facet are responsible for the observed peripheral material accumulation. In the case of AIN NWs under investigation, a similar behaviour is expected. However, the higher (1-100) surface diffusion barrier of AIN adatoms along the c-axis [73] compared to Ga favours the formation of an AIN shell around the GaN core, as seen in the lower part of figure 10c. In spite of this limited diffusion along the side walls correlated with a higher nucleation probability, it was estimated that about $60 \%$ of Al adatoms diffusing along the sidewall indeed reach the NW top [74] ]. Due to the higher (000-1) surface diffusion barrier of Al on AIN compared to Ga [73], the Al diffusion length on (000-1) AIN is even smaller than for Ga on (000-1) GaN so that the nucleation probability in the periphery of the NW top is expected to be even more marked in the case of AIN than in the case of GaN.

If now turning ourselves to the effect of $\mathrm{Mg}$, it was established that $\mathrm{Si}$ and $\mathrm{Mg}$ dopants dramatically affect the growth of GaN NWs. Whatever the dopant, the diameter 
was found to increase while the axial growth rate decreased, a behaviour assigned to the increased nucleation probability on the side walls, particularly marked in the case of $\mathrm{Mg}$ $[75,76]$. Accordingly, as schematically illustrated in figure 11 , the combination of Al diffusion along the sidewalls toward the NW top with the increased nucleation probability in presence of $\mathrm{Mg}$ and the very short $\mathrm{Al}(000-1)$ surface diffusion length provide the ingredients to account for the formation of the AIN nanotubes, which once initiated is made even more favourable due to the shadow effect preventing the Al flux to reach the (000-1) top surface. As shown in figure 10c, the diameter of the GaN NW base is about $50 \mathrm{~nm}$, while the diameter at the top of the AIN NT is around $235 \mathrm{~nm}$. Most of the enlargement is observed to take place during the growth of the AIN NT section under exposure to the Mg flux, consistent with the above considerations. According to the very short Al diffusion length on (000-1) AIN surface, it is reasonable to assume that the single nucleation event regime is unlikely in the elongation process of undoped or doped AIN NWs. Thus, the rapid enlargement of the AIN NWs under Mg flux combined to the enhanced Al diffusion towards the top of the side walls is expected to further promote both the peripheral nucleation of AIN and a fast switching to the NT regime illustrated in figure 11.

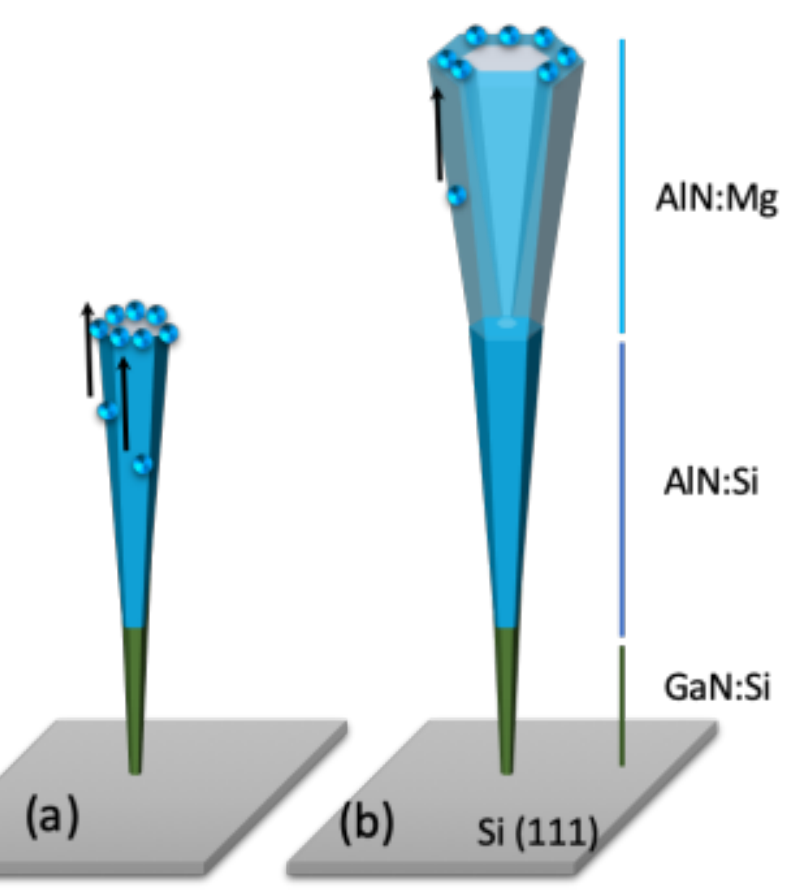

Figure 11: (a) Nucleation step of the AIN NT section under exposure to a Mg flux associated with an increased nucleation probability on the side walls. (b) Self-sustained steady state growth of the NTs 
In a recent article, Bolshakov et al. have reported the Si dopant-stimulated formation of GaN nanotubes [66]. As a matter of fact, Si doping of GaN is associated with tensile strain building up [77]. The relaxation of this strain obeys a specific mechanism in the case of GaN NWs, namely the formation of a Si-rich shell above the Si solubility limit, associated with a morphology change [78]. The increase in nucleation probability on the sidewall was assigned by Bolshakov et al. to the accumulation of Si dopant in the outer part of the NW for high enough doping level [66]. The situation is different in the case of AIN NWs under scrutiny. ptype doping of such NWs with Mg could be recently achieved by using In as a co-dopant [79]. However, in absence of In, the amount of incorporated Mg atoms in AIN NWs was negligible, which was assigned to both the size of $\mathrm{Mg}$ and its tendency to segregate on the surface, similar to what is found in the case of GaN layers [80]. In absence of In co-doping when growing the upper AIN NW section in the present case, it can be concluded that the enhanced lateral nucleation probability responsible for the formation of the AIN nanotubes is indeed associated with the surface segregation of $\mathrm{Mg}$. Studies on the $\mathrm{Mg}$ doping of GaN NWs have put in evidence a preferential Mg incorporation through the sidewalls, due to the stabilization of a $\mathrm{Mg}-\mathrm{H}$ reconstruction on the $\mathrm{m}$-plane $[81,82]$. The present data indeed suggest that a similar mechanism could be effective in the case of AIN, with the consequence of stabilizing the Mg-rich outer layer of AIN and enhancing the sidewall nucleation probability.

The diffusion of metallic species on the m-plane was studied for both GaN and AIN $[73,83]$. In both cases, the diffusion was found to be very anisotropic, with a surface diffusion barrier significantly lower in the [11-20] direction compared to [0001]. This is particularly true in the case of AIN, for which the surface diffusion barrier is $0.11 \mathrm{eV}$ along [11-20] whereas it is $2.79 \mathrm{eV}$ along [0001]. However, if considering the a-plane, this surface diffusion barrier along [0001] direction is lowered down to $0.56 \mathrm{eV}$ [73]. Similar to GaN NWs, AIN ones exhibit m-plane sidewalls, consistent with a lower (1-100) surface energy with respect to (11-20) [84]. Nevertheless, the appearance of (11-20) facets at the intersections of adjacent (1-100) planes [85] suggests that the Al diffusion channel from the sidewalls to the top is more likely on the (11-20) narrow facet connecting (1-100) planes facet, which exhibits a lower surface diffusion barrier along the [0001] direction. As the very short Al diffusion length on (000-1) top facet prevents homogenization of $\mathrm{Al}$ adatom 
coverage on top, nucleation is expected to randomly occur on different spots corresponding to the crossing points of (1-100) facets, eventually leading to a rough upper part of the AIN nanotubes, consistent with experimental observations.

\section{Conclusion}

To conclude, it was shown that the morphology of catalyst-free III-nitride nanowires grown by PAMBE is governed to a large extent by metal adatom diffusion along the side walls, in agreement with the pioneering observation of Debnath et al. [11]. Furthermore, for GaN NWs, it was shown that depending on the metal/nitrogen flux ratio value, the Ga adatom diffusion length could be tuned in order to be smaller or longer than the NW upper surface diameter. Experimental data are consistent with the presence of a diffusion barrier affecting the metal diffusion from the side walls to the top and, conversely, from the top to the sidewalls. Due to the presence of this diffusion barrier, GaN nucleation on top tends to be more favourable close to the periphery, which leads to peripheral bulging of the GaN NW upper surface. Associated with this bulging, the existence of steps is identified as the origin of spontaneous superlattice formation, characteristic of InGaN and AIGaN NWs. Consequently to spontaneous chemical ordering, the systematic carrier localization observed in AIGaN NWs makes them particularly attractive for opto-electronic applications. In addition, engineering metal diffusion along the side walls using surfactants opens a path for controlling the morphology of nitride-based nano-objects. Eventually, in the case of AIN $\mathrm{NWs}$, the enhanced diffusion of $\mathrm{Al}$ adatom along the side walls under the influence of $\mathrm{Mg}$ is shown to trigger the formation of AIN nanotubes, supporting the major role of kinetics in determining the growth mode of these III-N elongated nano-objects.

\section{Acknowledgments}

We benefitted from the access to the Nano characterization platform (PFNC) in CEA Minatec-Grenoble in collaboration with the IRIG/LEMMA group. AC and NG acknowledge financial support from project ENE2016-79282-C5-3-R cofinanced by the Spanish MICINN and FEDER funds and PROMETEO/2018/123 from Generalitat Valenciana. 


\section{References}

[1] Glas F 2006 Critical dimensions for the plastic relaxation of strained axial heterostructures in free-standing nanowires Phys. Rev. B - Condens. Matter Mater. Phys. 74 2-5

[2] Kikuchi A, Kawai M, Tada M and Kishino K 2004 InGaN/GaN multiple quantum disk nanocolumn light-emitting diodes grown on (111) Si substrate Japanese J. Appl. Physics, Part 2 Lett. 43 L1524

[3] Wang R, Nguyen H P T, Connie A T, Lee J, Shih I and Mi Z 2014 Color-tunable, phosphor-free InGaN nanowire light-emitting diode arrays monolithically integrated on silicon Opt. Express 22 A1768

[4] Huang Y, Duan X, Cui Y and Lieber C M 2002 Gallium Nitride Nanowire Nanodevices Nano Lett. 2 101-4

[5] Williams E H, Davydov A V., Oleshko V P, Steffens K L, Levina I, Lin N J, Bertness K A, Manocchi A K, Schreifels J A and Rao M V. 2014 Solution-based functionalization of galliumnitride nanowires for protein sensor development Surf. Sci. 627 23-8

[6] Zhou Z, Zhao J, Chen Y, Schleyer P V R and Chen Z 2007 Energetics and electronic structures of AIN nanotubes/wires and their potential application as ammonia sensors Nanotechnology 18424023

[7] Beheshtian J, Baei M T, Bagheri Z and Peyghan A A 2012 AIN nanotube as a potential electronic sensor for nitrogen dioxide Microelectronics J. 43 452-5

[8] Wagner R S and Ellis W C 1964 Vapor-liquid-solid mechanism of single crystal growth Appl. Phys. Lett. 4 89-90

[9] Yoshizawa M, Kikuchi A, Mori M, Fujita N and Kishino K 1997 Growth of self-organized GaN nanostructures on Al203(0001) by RF-radical source molecular beam epitaxy Japanese J. Appl. Physics, Part 2 Lett. 36 L459

[10] Sanchez-Garcia M A, Calleja E, Monroy E, Sanchez F J, Calle F, Muñoz E and Beresford $\mathrm{R} 1998$ The effect of the III/V ratio and substrate temperature on the morphology and properties of GaN- and AIN-layers grown by molecular beam epitaxy on $\mathrm{Si}\left(\begin{array}{lll}1 & 1 & 1\end{array}\right) \mathrm{J}$. Cryst. Growth 183 23-30

[11] Debnath R K, Meijers R, Richter T, Stoica T, Calarco R and Lüth H 2007 Mechanism of molecular beam epitaxy growth of GaN nanowires on Si(111) Appl. Phys. Lett. 90 1-4

[12] Calarco R, Meijers R J, Debnath R K, Stoical T, Sutter E and Lüth H 2007 Nucleation and growth of GaN nanowires on $\mathrm{Si}(111)$ performed by molecular beam epitaxy Nano Lett. $72248-51$

[13] Stoica T, Sutter E, Meijers R J, Debnath R K, Calarco R, Lüth H and Grützmacher D 2008 Interface and wetting layer effect on the catalyst-free nucleation and growth of GaN nanowires Small 4 751-4 
[14] Park Y, Jahangir S, Park Y, Bhattacharya P and Heo J 2015 InGaN/GaN nanowires grown on $\mathrm{SiO} 2$ and light emitting diodes with low turn on voltages Opt. Express 23 A650

[15] van Treeck D, Calabrese G, Goertz J J W, Kaganer V M, Brandt O, Fernández-Garrido S and Geelhaar $L 2018$ Self-assembled formation of long, thin, and uncoalesced GaN nanowires on crystalline TiN films Nano Res. 11 565-76

[16] Kumaresan V, Largeau L, Madouri A, Glas F, Zhang H, Oehler F, Cavanna A, Babichev A, Travers L, Gogneau N, Tchernycheva M and Harmand J C 2016 Epitaxy of GaN Nanowires on Graphene Nano Lett. 16 4895-902

[17] Fernández-Garrido S, Ramsteiner $M$, Gao G, Galves L A, Sharma B, Corfdir $P$, Calabrese G, De Souza Schiaber Z, Pfüller C, Trampert A, Lopes J M J, Brandt O and Geelhaar L 2017 Molecular Beam Epitaxy of GaN Nanowires on Epitaxial Graphene Nano Lett. 17 5213-21

[18] Schuster F, Furtmayr F, Zamani R, Magén C, Morante J R, Arbiol J, Garrido J A and Stutzmann M 2012 Self-assembled GaN nanowires on diamond Nano Lett. 12 2199204

[19] Sergent S, Damilano B, Vézian S, Chenot S, Tsuchizawa T and Notomi M 2020 Lasing up to $380 \mathrm{~K}$ in a sublimated GaN nanowire Appl. Phys. Lett. 116223101

[20] Consonni V, Knelangen M, Geelhaar L, Trampert A and Riechert H 2010 Nucleation mechanisms of epitaxial GaN nanowires: Origin of their self-induced formation and initial radius Phys. Rev. B - Condens. Matter Mater. Phys. 81 1-10

[21] Largeau L, Galopin E, Gogneau N, Travers L, Glas F and Harmand J C 2012 N-polar GaN nanowires seeded by Al droplets on Si(111) Cryst. Growth Des. 12 2724-9

[22] Auzelle T, Haas B, Minj A, Bougerol C, Rouvière J-L, Cros A, Colchero J and Daudin B 2015 The influence of AIN buffer over the polarity and the nucleation of self-organized GaN nanowires J. Appl. Phys. 117245303

[23] Songmuang R, Landré O and Daudin B 2007 From nucleation to growth of catalystfree GaN nanowires on thin AIN buffer layer Appl. Phys. Lett. 91251902

[24] Glas F, Harmand J C and Patriarche G 2007 Why does wurtzite form in nanowires of III-V zinc blende semiconductors? Phys. Rev. Lett. 99 3-6

[25] Glas F, Ramdani M R, Patriarche G and Harmand J C 2013 Predictive modeling of selfcatalyzed III-V nanowire growth Phys. Rev. B - Condens. Matter Mater. Phys. 88 1-14

[26] Gamalski A D, Ducati C and Hofmann S 2011 Cyclic supersaturation and triple phase boundary dynamics in germanium nanowire growth J. Phys. Chem. C 115 4413-7

[27] Daudin B, Rouvière J L and Arlery M 1996 Polarity determination of GaN films by ion channeling and convergent beam electron diffraction Appl. Phys. Lett. 69 2480-2

[28] Rouvière J L, Weyher J L, Seelmann-Eggebert M and Porowski S 1998 Polarity determination for GaN films grown on (0001) sapphire and high-pressure-grown GaN 
single crystals Appl. Phys. Lett. 73 668-70

[29] Zywietz T, Neugebauer J and Scheffler M 1998 Adatom diffusion at GaN (0001) and (0001) surfaces Appl. Phys. Lett. 73 487-9

[30] Adelmann C, Brault J, Jalabert D, Gentile P, Mariette H, Mula G and Daudin B 2002 Dynamically stable gallium surface coverages during plasma-assisted molecular-beam epitaxy of (0001) GaN J. Appl. Phys. 91 9638-45

[31] Northrup J E and Neugebauer J 2000 Structure of GaN(0001): The laterally contracted Ga bilayer model Phys. Rev. B - Condens. Matter Mater. Phys. 61 9932-5

[32] Monroy E, Sarigiannidou E, Fossard F, Gogneau N, Bellet-Amalric E, Rouvière J L, Monnoye S, Mank H and Daudin B 2004 Growth kinetics of N-face polarity GaN by plasma-assisted molecular-beam epitaxy Appl. Phys. Lett. 84 3684-6

[33] Li H, Geelhaar L, Riechert H and Draxl C 2015 Computing Equilibrium Shapes of Wurtzite Crystals: The Example of GaN Phys. Rev. Lett. 115 1-5

[34] Kishino K, Sekiguchi H and Kikuchi A 2009 Improved Ti-mask selective-area growth (SAG) by rf-plasma-assisted molecular beam epitaxy demonstrating extremely uniform GaN nanocolumn arrays J. Cryst. Growth 311 2063-8

[35] Mata R, Hestroffer K, Budagosky J, Cros A, Bougerol C, Renevier H and Daudin B 2011 Nucleation of GaN nanowires grown by plasma-assisted molecular beam epitaxy: The effect of temperature J. Cryst. Growth 334 177-80

[36] Gruart M, Jacopin G and Daudin B 2019 Role of Ga Surface Diffusion in the Elongation Mechanism and Optical Properties of Catalyst-Free GaN Nanowires Grown by Molecular Beam Epitaxy Nano Lett. 19 4250-6

[37] Fernández-Garrido S, Kaganer V M, Sabelfeld K K, Gotschke T, Grandal J, Calleja E, Geelhaar L and Brandt O 2013 Self-regulated radius of spontaneously formed GaN nanowires in molecular beam epitaxy Nano Lett. 13 3274-80

[38] Kouno T, Kishino K and Sakai M 2011 Lasing action on whispering gallery mode of selforganized GaN hexagonal microdisk crystal fabricated by RF-plasma-assisted molecular beam epitaxy IEEE J. Quantum Electron. 47 1565-70

[39] Kouno T and Kishino K 2012 Well-arranged novel InGaN hexagonal nanoplates at the tops of nitrogen-polarity GaN nanocolumn arrays AIP Adv. 2012140

[40] Kouno T, Sakai M, Kishino K and Hara K 2014 Hexagonal GaN microdisk with wurtzite/zinc-blende GaN crystal phase nano-heterostructures and high quality zincblende GaN crystal layer Jpn. J. Appl. Phys. 53068001

[41] Zhang X, Haas B, Rouvière J L, Robin E and Daudin B 2016 Growth mechanism of InGaN nano-umbrellas Nanotechnology 27455603

[42] Bertness K A, Roshko A, Sanford N A, Barker J M and Davydov A V. 2006 Spontaneously grown GaN and AlGaN nanowires J. Cryst. Growth 287 522-7 
[43] Feenstra R M, Chen H, Ramachandran V, Lee C D, Smith A R, Northrup J E, Zywietz T, Neugebauer J and Greve D W 2000 Surface Morphology of GaN Surfaces During Molecular Beam Epitaxy Surf. Rev. Lett. 07 601-6

[44] Pierret A, Bougerol C, Murcia-Mascaros S, Cros A, Renevier H, Gayral B and Daudin B 2013 Growth, structural and optical properties of AlGaN nanowires in the whole composition range Nanotechnology 24115704

[45] Robinson R D, Sadtler B, Demchenko D O, Erdonmez C K, Wang L W and Alivisatos A P 2007 Spontaneous superlattice formation in nanorods through partial cation exchange Science (80-. ). 317 355-8

[46] Gogneau N, Jalabert D, Monroy E, Sarigiannidou E, Rouvière J L, Shibata T, Tanaka M, Gerard J M and Daudin B 2004 Influence of AIN overgrowth on structural properties of GaN quantum wells and quantum dots grown by plasma-assisted molecular beam epitaxy J. Appl. Phys. 96 1104-10

[47] Zhao S, Woo S Y, Sadaf S M, Wu Y, Pofelski A, Laleyan D A, Rashid R T, Wang Y, Botton $G$ A and Mi Z 2016 Molecular beam epitaxy growth of Al-rich AIGaN nanowires for deep ultraviolet optoelectronics APL Mater. 4086115

[48] Pierret A, Bougerol C, Hertog M den, Gayral B, Kociak M, Renevier H and Daudin B 2013 Structural and optical properties of Alx Ga1-xN nanowires Phys. Status Solidi Rapid Res. Lett. 7 868-73

[49] Himwas C, Hertog M Den, Dang LS, Monroy E, Songmuang R, Himwas C, Hertog M Den, Dang L S, Monroy E and Songmuang R 2016 Alloy inhomogeneity and carrier localization in AIGaN sections and AIGaN / AIN nanodisks in nanowires with 240-350 nm emission 105241908

[50] Behbehani M K, Piner E L, Liu S X, El-Masry N A and Bedair S M 1999 Phase separation and ordering coexisting in InxGa1-xN grown by metal organic chemical vapor deposition Appl. Phys. Lett. 75 2202-4

[51] El-Masry N A, Behbehani M K, LeBoeuf S F, Aumer M E, Roberts J C and Bedair S M 2001 Self-assembled AllnGaN quaternary superlattice structures Appl. Phys. Lett. 79 $1616-8$

[52] Ruterana P, De Saint Jores G, Laügt M, Omnes F and Bellet-Amalric E 2001 Evidence for multiple chemical ordering in AIGaN grown by metalorganic chemical vapor deposition Appl. Phys. Lett. 78 344-6

[53] Iliopoulos E, Ludwig K F, Moustakas T D and Chu S N G 2001 Chemical ordering in AlGaN alloys grown by molecular beam epitaxy Appl. Phys. Lett. 78 463-5

[54] Strittmatter A, Reissmann L, Bimberg D, Veit P and Krost A 2002 Spontaneous superlattice formation in AIGaN layers grown by MOCVD on Si(111)-substrates Phys. Status Solidi Basic Res. 234 722-5

[55] Pakula K, Bozek R, Baranowski J M and Jasinski J 2005 Spontaneous superlattice formation in MOVPE growth of AIGaN Phys. Status Solidi C Conf. 2 1073-6 
[56] Northrup J E, Romano L T and Neugebauer J 1999 Surface energetics, pit formation, and chemical ordering in InGaN alloys Appl. Phys. Lett. 74 2319-21

[57] Lymperakis L, Abu-Farsakh H, Marquardt O, Hickel T and Neugebauer J 2011 Theoretical modeling of growth processes, extended defects, and electronic properties of III-nitride semiconductor nanostructures Phys. Status Solidi Basic Res. 248 1837-52

[58] Albrecht M, Lymperakis L, Neugebauer J, Northrup J E, Kirste L, Leroux M, Grzegory I, Porowski S and Strunk H P 2005 Chemically ordered Al xGa 1-xN alloys: Spontaneous formation of natural quantum wells Phys. Rev. B - Condens. Matter Mater. Phys. 71 15

[59] Pierret A, Bougerol C, Gayral B, Kociak M and Daudin B 2013 Probing alloy composition gradient and nanometer-scale carrier localization in single AIGaN nanowires by nanocathodoluminescence Nanotechnology 24305703

[60] Okuno H, Rouvìre J L, Jouneau P H, Bayle-Guillemaud P and Daudin B 2010 Visualization of Tm dopant atoms diffused out of GaN quantum dots Appl. Phys. Lett. $961-4$

[61] Gómez-Gómez M, Garro N, Segura-Ruiz J, Martinez-Criado G, Cantarero A, Mengistu H T, García-Cristóbal A, Murcia-Mascarós S, Denker C, Malindretos J and Rizzi A 2014 Spontaneous core-shell elemental distribution in In-rich InxGa1-xN nanowires grown by molecular beam epitaxy Nanotechnology 25075705

[62] Zhang $X$, Lourenço-Martins $H$, Meuret $S$, Kociak $M$, Haas $B$, Rouvière J L, Jouneau P H, Bougerol C, Auzelle T, Jalabert D, Biquard X, Gayral B and Daudin B 2016 InGaN nanowires with high InN molar fraction: Growth, structural and optical properties Nanotechnology 27195704

[63] Belloeil M, Proietti M G, Renevier H and Daudin B 2020 Nanoscale x-ray investigation of composition fluctuations in AIGaN nanowires Nanotechnology 31375709

[64] Belloeil M, Gayral B and Daudin B 2016 Quantum Dot-Like Behavior of Compositional Fluctuations in AIGaN Nanowires Nano Lett. 16 960-6

[65] Goldberger J, He R, Zhang Y, Lee S, Yan H, Choi H J and Yang P 2003 Single-crystal gallium nitride nanotubes Nature 422 599-602

[66] Bolshakov A D, Mozharov A M, Sapunov G A, Shtrom I V., Sibirev N V., Fedorov V V., Ubyivovk E V., Tchernycheva M, Cirlin G E and Mukhin I S 2018 Dopant-stimulated growth of GaN nanotube-like nanostructures on Si(111) by molecular beam epitaxy Beilstein J. Nanotechnol. 9 146-54

[67] Schuster F, Hetzl M, Weiszer S, Garrido J A, De La Mata M, Magen C, Arbiol J and Stutzmann M 2015 Position-controlled growth of GaN nanowires and nanotubes on diamond by molecular beam epitaxy Nano Lett. 15 1773-9

[68] Hemmingsson C, Pozina G, Khromov S and Monemar B 2011 Growth of GaN nanotubes by halide vapor phase epitaxy Nanotechnology 22085602 
[69] Durand C, Carlin J F, Bougerol C, Gayral B, Salomon D, Barnes J P, Eymery J, Butté R and Grandjean N 2017 Thin-Wall GaN/InAIN Multiple Quantum Well Tubes Nano Lett. 17 3347-55

[70] Wu Q, Hu Z, Wang X, Lu Y, Chen X, Xu H and Chen Y 2003 Synthesis and characterization of faceted hexagonal aluminum nitride nanotubes J. Am. Chem. Soc. $12510176-7$

[71] Landré O, Fellmann V, Jaffrennou P, Bougerol C, Renevier H, Cros A and Daudin B 2010 Molecular beam epitaxy growth and optical properties of AIN nanowires Appl. Phys. Lett. 96061912

[72] Arbiol J, Estradé S, Prades J D, Cirera A, Furtmayr F, Stark C, Laufer A, Stutzmann M, Eickhoff M, Gass M H, Bleloch A L, Peiró F and Morante J R 2009 Triple-twin domains in $\mathrm{Mg}$ doped GaN wurtzite nanowires: Structural and electronic properties of this zincblende-like stacking Nanotechnology 20145704

[73] Jindal V and Shahedipour-Sandvik F 2009 Density functional theoretical study of surface structure and adatom kinetics for wurtzite AIN J. Appl. Phys. 105084902

[74] Hestroffer K and Daudin B 2013 A geometrical model for the description of the AIN shell morphology in GaN-AIN core-shell nanowires J. Appl. Phys. 114244305

[75] Furtmayr F, Vielemeyer M, Stutzmann M, Arbiol J, Estrad́ S, Peir F, Morante J R and Eickhoff M 2008 Nucleation and growth of GaN nanorods on Si (111) surfaces by plasma-assisted molecular beam epitaxy - The influence of Si- and Mg-doping J. Appl. Phys. 104 1-7

[76] Sekiguchi H, Kato K, Tanaka J, Kikuchi A and Kishino K 2008 Ultraviolet GaN-based nanocolumn light-emitting diodes grown on n-(111) Si substrates by rf-plasmaassisted molecular beam epitaxy Phys. Status Solidi Appl. Mater. Sci. 205 1067-9

[77] Sánchez-Páramo J, Calleja J M, Sánchez-García M A and Calleja E 2001 Optical investigation of strain in Si-doped GaN films Appl. Phys. Lett. 78 4124-6

[78] Fang Z, Robin E, Rozas-Jiménez E, Cros A, Donatini F, Mollard N, Pernot J and Daudin B 2015 Si Donor Incorporation in GaN Nanowires Nano Lett. 15 6794-801

[79] Siladie A M, Jacopin G, Cros A, Garro N, Robin E, Caliste D, Pochet P, Donatini F, Pernot J and Daudin B $2019 \mathrm{Mg}$ and in Codoped p-type AIN Nanowires for pn Junction Realization Nano Lett. 19 8357-64

[80] Mula G, Daudin B, Adelmann C and Peyla P 2000 MBE growth of GaN films in presence of surfactants: The effect of Mg and Si Mater. Res. Soc. Symp. - Proc. 595

[81] Siladie A M, Amichi L, Mollard N, Mouton I, Bonef B, Bougerol C, Grenier A, Robin E, Jouneau P H, Garro N, Cros A and Daudin B 2018 Dopant radial inhomogeneity in Mgdoped GaN nanowires Nanotechnology 29255706

[82] Northrup J E 2008 Hydrogen and magnesium incorporation on c -plane and m -plane GaN surfaces Phys. Rev. B - Condens. Matter Mater. Phys. 77 1-7 
[83] Lymperakis L and Neugebauer J 2009 Large anisotropic adatom kinetics on nonpolar GaN surfaces: Consequences for surface morphologies and nanowire growth Phys. Rev. B - Condens. Matter Mater. Phys. 79 1-4

[84] Zhao M, Xia Y, Liu X, Tan Z, Huang B, Song C and Mei L 2006 First-principles calculations of AIN nanowires and nanotubes: Atomic structures, energetics, and surface states J. Phys. Chem. B $1108764-8$

[85] Arbiol J, Magen C, Becker P, Jacopin G, Chernikov A, Schäfer S, Furtmayr F, Tchernycheva M, Rigutti L, Teubert J, Chatterjee S, Morante J R and Eickhoff M 2012 Self-assembled GaN quantum wires on GaN/AIN nanowire templates Nanoscale 4 7517-24 